\title{
Design and development of an e-nose system for the diagnosis of pulmonary diseases
}

\author{
V.A. BINSON*, M. SUBRAMONIAM \\ Department of Electronics Engineering, Sathyabama Institute of Science \& Technology, Chennai, India.
}

\begin{abstract}
Purpose: The aim of this paper was to discuss the design and development of an innovative e-nose system which can detect respiratory ailments by detecting the Volatile Organic Compounds (VOCs) in the expelled breath. In addition to nitrogen, oxygen, and carbon dioxide, the expelled breath contains several VOCs, some of which are indicative of lung-related conditions and can differentiate healthy controls from people affected with pulmonary diseases. Methods: This work detailed the sensor selection process, the assembly of the sensors into a sensor array, the design and implementation of the circuit, sampling methods, and an algorithm for data analysis. The clinical feasibility of the system was checked in 27 lung cancer patients, 22 chronic obstructive pulmonary disease (COPD) patients, and 39 healthy controls including smokers and non-smokers. Results: The classification model developed using the support vector machine (SVM) was able to provide accuracy, sensitivity, and specificity of $88.79,89.58$ and $88.23 \%$, respectively for lung cancer, and $78.70,72.50$ and $82.35 \%$, respectively for COPD. Conclusions: The sensor array system developed with TGS gas sensors was non-invasive, low cost, and gave a rapid response. It has been demonstrated that the VOC profiles of patients with pulmonary diseases and healthy controls are different, hence, the e-nose system can be used as a potential diagnostic device for patients with lung diseases.
\end{abstract}

Key words: breath analysis, volatile organic compounds, MOS sensors, lung cancer, COPD

\section{Introduction}

Human exhaled breath contains various VOCs (volatile organic compounds) other than nitrogen, oxygen, and carbon dioxide in lower concentrations. It has been identified that the exhaled breath of humans consists of more than 1000 VOC gases, a majority of which are of exogenous origin. Most VOCs are believed to reflect the organic processes that happen inside the human body at the tissue level, such as oxidative stress and inflammation. However, detected can be also the exogenous VOCs which indicate the pollution, radiation, and different kind of smokes such as from cigarettes, vehicles, factories, etc. [14]. The pathophysiological and physiological conditions of humans can be identified by the detection of exhaled breath volatile organic compounds [1]. These VOCs are used as biomarkers for various respiratory diseases such as chronic bronchitis, lung cancer, asthma, pneumonia, chronic obstructive pulmonary disease [COPD], etc. For example, the exhaled breath of lung cancer patients contains higher concentrations of propanol, acetone, hexanal, and toluene, and the exhaled breath of pneumonia patients contains high concentrations of acetone, nonanal, ethanol, and heptane than the healthy controls. The concentrations of 2-pentanone, $n$-butane, 4-heptanone, and cyclohexanone are high in COPD patients than healthy people. Hence, exhaled breath analysis is a promising diagnostic tool as they are non-invasive and fast.

A typical gas sensor used for VOC analysis should be highly responsive, selective, and stable. In practical applications, a cost-effective effortless fabrication is expected. To obtain these specifications, different types of gas sensors with various means of sensing principles, e.g., solid-state electrochemical sensor (SSES), metal oxide semiconductor sensor (MOS), surface acoustic

\footnotetext{
* Corresponding author: V.A. Binson, Department of Electronics Engineering, Sathyabama Institute of Science and Technology, Jeppiaar Nagar, Rajiv Gandhi Salai, Chennai - 600 119. Tamilnadu, India. Phone: +91-9526738829, e-mail: binsonvsabraham@gmail.com

Received: October 17th, 2020

Accepted for publication: December 15th, 2020
} 
wave sensor (SAW), photoionization detector sensor (PID), quartz crystal microbalance sensor (QCM), etc have been developed [18]. The metal oxide semiconductor transduction principle is based on the conductivity change of the sensing element in response to the application of gases. These types of sensors are developed by exposing the sensing layer directly to the gaseous mixture. The variation in conductivity happens as the sensing layer is exposed to the analytes and, in turn, varies the chemical and physical properties of the primary sensing element at higher temperatures [10].

An exhaled breath measuring system uses different types of sensors such as metal oxide semiconductor gas (VOC gas) sensors which were developed for a wide variety of applications comprising gas leak detection systems and air quality monitors [18], and disease diagnosis in humans [4], [9]. There is a chance of the presence of possible gases that interfere, so the selection of gas sensors must be examined accurately in all applications. By the addition of a noble metal catalyst, the selectivity of metal oxide gas sensors could be controlled. The sensor array could be analyzed using numerous statistical methods including linear discriminant analysis and principal component analysis [4], [15]. The VOCs associated with some diseases are in parts per billion (ppb) ranges instead of parts per million (ppm) ranges in exhaled breath. This low concentration of VOCs makes it difficult for some MOS sensors to make perfect measurements. Pasini et al. [23] developed a gas sensor array system with four MOS sensors for the detection of volatile organic compounds in yeast strains, yeast cell suspension, and chemically induced cells. The system was able to differentiate induced cells with non-induced cells. Similarly, Li et al. [19] developed a lung cancer screening device with a sensor array consisting of eight MOS gas sensors and analyzed the breath samples of 52 volunteers. They used five feature selection algorithms and two classifiers to distinguish between lung cancer patients, controls, and volunteers with other respiratory diseases.

Lung cancer is the leading cause of cancer-related death in men and the second leading cause of cancer-related death in women globally. The trends and prevalence of lung cancer vary based on gender, age, smoking status, ethnicity, economic status, and geographical locations. Most of the patients affected with lung cancer are diagnosed at a late stage and the curative treatment is impossible, hence, the survival rate reduces. In India, the major cause of lung cancer is tobacco smoking both as cigarette and beedi. COPD is also a high-risk pulmonary disease with an increased mortality rate globally. Like lung cancer, cigarette smoking is the primary risk factor for COPD and the other risk factors include genetic factors, air pollutants, occupational health hazards, age, asthma, and infections. COPD is a preventable and treatable disease [20]. Lung cancer and COPD can be diagnosed with different MOS sensor arrays using the breath analysis technique [6], [19].

Research is being carried out in the breath analysis using e-nose for the possible prediction of respiratory diseases. E-nose technology is used to differenciate patients with various lung diseases, such as cystic fibrosis, asthma, COPD, chronic bronchitis, and lung cancer, and healthy controls. This method is also used in the diagnosis of various other diseases, such as prostate cancer, urinary tract infections, uraemia, gastric cancer, diabetes, asbestos-related diseases, colorectal cancer, bacterial vaginosis, etc. [29].

Pavlou et al. [24] analyzed the volatile organic compounds in the human urine samples with an e-nose consisting of fourteen conducting polymer sensors. The developed system was able to distinguish Escherichia coli infected, Staphylococcus spp, Proteus spp, and normal urine. In another study using electronic nose and Field Asymmetric Ion Mobility Spectrometer developed by Owlstone Medicals, it was able to differentiate ulcerative colitis, Bile acid diarrhoea, and healthy controls by analyzing the VOCs in the urine sample [7]. A low-cost e-nose equipped with six MOS sensors at the University of Pamplona, Colombia was used for the exhaled breath VOC analysis of patients with COPD and healthy controls [28]. Roine et al. [25] were able to differentiate benign prostatic hyperplasia from prostate cancer by the VOC analysis of urine samples with ChemPro 100-eNose. The sensitivity and specificity of the system were $78 \%$ and $67 \%$, respectively, for a study conducted on 50 prostate cancer patients and 24 benign prostatic hyperplasia patients. The e-nose system developed at the University of Rome Tor Vergata using eight QCM sensors was used to analyze the exhaled VOCs of lung cancer patients, patients with other respiratory diseases, and healthy controls [8]. The classification model was developed using Partial Least Squares Discriminant analysis and a sensitivity of $92.8 \%$ and $78.6 \%$ was achieved for lung cancer patients and patients with other lung diseases, respectively.

In this work, we have developed a system using metal oxide semiconductor gas sensors to detect the presence of volatile organic compounds present in the expelled breath of humans and, thereby, differentiate lung cancer from healthy controls and COPD from healthy controls. The VOC profile is a breath signature that varies from person to person and can be used 
to analyze the lung conditions and diseases. Here, we have developed a low-cost e-nose system with five MOS gas sensors, an Arduino Uno Rev3 developer board, and a machine learning algorithm for data analysis. The breath samples of 79 study subjects were analyzed using e-nose to understand their lung conditions.

For this, we have utilized five MOS gas sensors TGS 2600, TGS 2610, TGS 2620, TGS 822, and TGS 826 to detect the presence of VOCs in the exhaled breath. Each of these TGS gas sensors generates a response to various volatile organic compounds to which they are exposed but generates another response to

\section{Materials and methods}

\subsection{Gas sensors}

Five commercially available semiconductor metal oxide gas sensors developed by Figaro USA, Inc. are used for the development of sensor array. The gas sensors used are TGS 2600, TGS 2610, TGS 2620, TGS 822 , and TGS 826. The details of sensors are depicted in Table 1.

Table 1. Details of TGS sensors

\begin{tabular}{|l|l|c|c|}
\hline \multicolumn{1}{|c|}{ Model number } & \multicolumn{1}{|c|}{ Detected compounds } & Range [ppm] & Sensing principle \\
\hline TGS 2600 & Methane, Isobutane, Ethanol, and Carbon Monoxide & $1-100$ & MOS type \\
\hline TGS 2610 & Ethanol, Methane, Propane, and Isobutane & $300-10000$ & MOS type \\
\hline TGS 2620 & Carbon Monoxide, Ethanol, Isobutane, and Methane & $50-5000$ & MOS type \\
\hline TGS 822 & Acetone, Ethanol, Benzene, and Methane & $50-5000$ & MOS type \\
\hline TGS 826 & Ammonia, Ethanol, Hydrogen, and Isobutane & $30-300$ & MOS type \\
\hline
\end{tabular}

a gas that is more sensitive to that sensor. Thus, rather than recognizing a single VOC from human breath these sensors are used to generate a "breath fingerprint" for a combination of VOCs. By reviewing various papers related to the diagnosis of lung cancer [6], [19], [21] and COPD [5], [12], using TGS sensors, we have decided to use five sensors in our study maintaining better system accuracy. In special applications like disease diagnosis using breath analysis, environmental pollution detection, and quality analysis of food and beverages, the sensing characteristics of metal oxide gas sensors should be increased to fulfil the demands. The design of a MOS-based gas sensor circuit for these applications posses numerous challenges including the selection of gas sensors that gives good selectivity, quick response, a small range of detection, and low power consumption. The sensing property of a MOS sensor depends on the morphological structure, heterointerface structure, and defect structure. The novelty of this work is the selection of a minimum number of MOS sensors that can give accurate results in the prediction of respiratory diseases like lung cancer and COPD. All the previous studies based on VOC analysis of expelled breath using e-nose for COPD [26], [28] and lung cancer [6], [21], [22] diagnosis used patients and healthy controls with entirely different age groups. In this work, we have carefully selected the healthy controls and patients of almost similar age groups, even if it was a tough task.
The sensing elements of all the Figaro gas sensors used in this work are made up of a metal oxide semiconductor layer whose conductivity is very low in clean air. When the detectable gases are present, the conductivity of the gas sensor increases depending on the gas concentration in the air. The change in conductivity can be converted to an electrical output signal with the help of a simple electrical circuit. All the TGS sensors used in this study are cross response sensors and not gas specific sensors. The response of these sensors to the same gas mixture is different and the response of the same sensor to the different gas mixture is also different. Instead of measuring a single compound (VOC) from exhaled breath, these sensors produce a voltage signal corresponding to the mixture of exhalation components.

\subsection{Arduino Uno Rev3}

Arduino Uno is an ATmega328P-based microcontroller board. The technical specifications of the board are shown in Table 2. A mechanism of resettable polyfuse is fixed in the Arduino Uno board which protects the USB port of our computer from short circuits or over current. The major difference between the Uno and its early edition boards, such as Arduino Nano, Mini, Mega, etc is that it uses programmed Atmega16U2 as USB to serial converter and does not use FTDI USB to serial driver chip. 
Table 2. Technical specifications of Arduino Uno

\begin{tabular}{|l|l|}
\hline Microcontroller & ATmega328P \\
\hline Voltage (Operating) & $5 \mathrm{~V}$ \\
\hline Voltage (Input) - Recommended & $7 \mathrm{~V}$ to $12 \mathrm{~V}$ \\
\hline Voltage (Input) - Limit & $6 \mathrm{~V}$ to $20 \mathrm{~V}$ \\
\hline Digital input/output pins & 14 (6 PWM outputs) \\
\hline PWM digital input/output pins & 6 \\
\hline Analog input pins & 6 \\
\hline DC Current per input/output pin & $20 \mathrm{~mA}$ \\
\hline Flash Memory & $32 \mathrm{~KB}$ \\
\hline Clock Speed & $16 \mathrm{MHz}$ \\
\hline
\end{tabular}

The designated 14 digital I/O pins can be used either as input or output using the Arduino functions and operate at a voltage of $5 \mathrm{~V}$. The analog inputs, from $\mathrm{A} 0$ to $\mathrm{A} 5$, provide 10 bits of resolution and they measure from $0 \mathrm{~V}$ to $5 \mathrm{~V}$. The communication facilities of Arduino Uno enable them to communicate with other Arduino boards, computer or microcontroller. The Arduino (IDE) Software comprises a serial monitor that admits simple text data set to communicate with the board in a full-duplex manner. Serial communication in any Uno digital pin is possible with a software serial library.

\subsection{Circuit details}

The circuit diagram of the e-nose system, which consists of the TGS sensors and Arduino Uno Rev3, is shown in Fig. 1. Corresponding to the variations in the input gas concentrations, an analog output voltage between $0 \mathrm{~V}$ and $5 \mathrm{~V}$ is produced in these TGS sensors and is fed to the Arduino which converts that to a digital signal. TGS 826 and TGS 822 are 6 pin gas sensors and the output is taken from the shorted fourth and sixth pin, whereas TGS 2600, TGS 2610, and TGS 2620 are 4 pin gas sensors and the output is taken from the second pin. Five analog input pins A0, A1, A2, A3, and $\mathrm{A} 4$ are used for data acquisition from sensors TGS 826, TGS 2610, TGS 2620, TGS 2600, and TGS 822 , respectively.

\subsection{The e-nose system}

The final assembly of the sensor array and the whole e-nose system is shown in Figs. $2 a$ and $2 b$, respectively. The sensors are powered with a $5 \mathrm{~V}$ DC

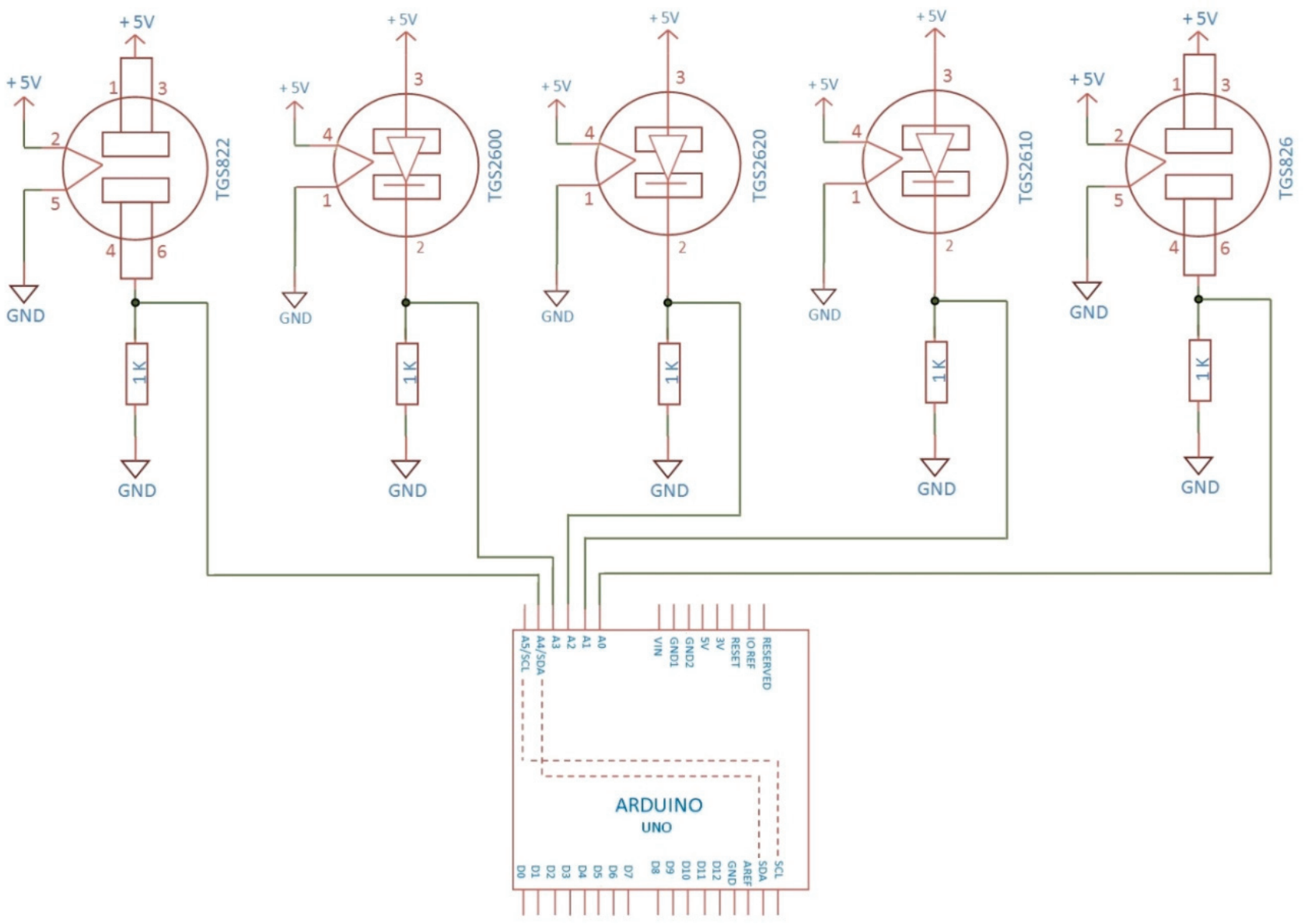

Fig. 1. Circuit diagram of the e-nose system 

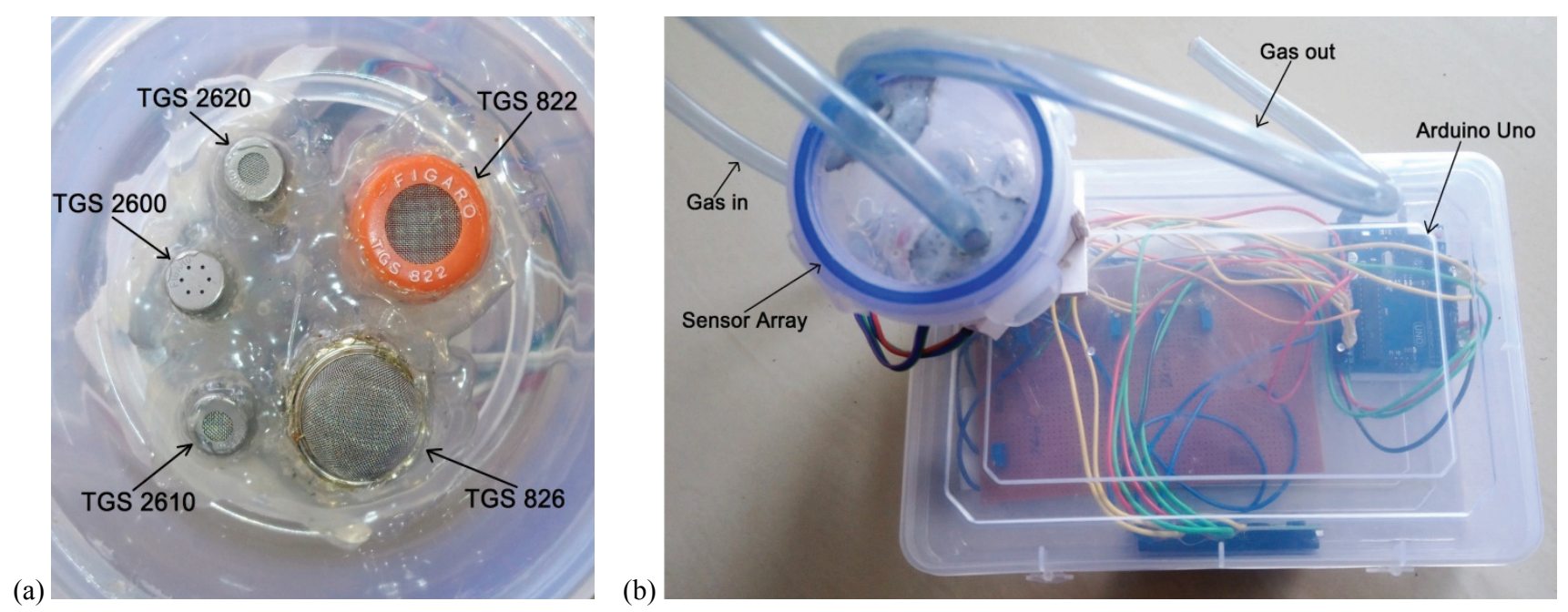

Fig. 2. (a) Sensor array, (b) the e-nose system

supply. We have connected two tubes with the sensor array system for gas in and gas out. The volunteers selected for the breath test were to blow air to the sensor system through the gas in a tube connected with the array. The output of the sensor array through the load resistor is fed through the Arduino board to a personal computer with machine learning algorithms.

\subsection{Study participants and data collection}

For this study, two sets of peoples were enrolled, one for the training set and another for the test set. 49 volunteers were selected for creating the training data set and the prediction model, and 39 volunteers to validate that model. The breath samples from the participants were collected using the e-nose system. All the study subjects were from the Kerala state in India. The study was approved by institutional ethical committee and written informed consent from all the volunteers under the study was collected. Out of the 49 participants in the training phase of the study, thirty-four were male and fifteen were female and in the validation phase, twenty-seven males and twelve females were selected. We included 22 healthy controls, 12 COPD patients, and 15 lung cancer patients for the training set and 17 healthy controls, 10 COPD patients, and 12 lung cancer patients for the test set. To understand the changes of breath VOCs for person to person, smokers and non-smokers were also included in the healthy group. We have also considered the age of the participants which varied from 44 to 72 years.

The MOS sensors used for the detection of breath VOCs were preheated for one hour at a constant voltage (heater voltage) of $5 \mathrm{~V}$. This step was repeated be- fore every sample collection. During sample collection, the participants were allowed to blow through the tube specified for air in. Reduction in drift and stability improvement is achieved with the sensor preheating before the sample collection and which, in turn, improved the repeatability of the measurements. All the five sensors produced a breath signature corresponding to the VOCs exposed to it, instead of giving a particular value for a specific VOC and we have measured the response for two minutes. We have taken four different samples from all the study subjects in a span of two months. The four samples were taken in the morning before breakfast (at $6.30 \mathrm{am}$ ), the morning after breakfast (at $10 \mathrm{am}$ ), noon after lunch (at $3 \mathrm{pm}$ ), and evening (at $6 \mathrm{pm}$ ). The volunteers were not allowed to smoke or consume alcohol on the test day and a day before the test and also food and water were restricted to two hours just before the test. The sensor outputs were almost consistent for all the five separate measurements, which show the repeatability of the sensor array in response to different VOCs is good.

\subsection{Data analysis}

The multi-dimensional sensor data produced by the five TGS gas sensors were given to the machine learning classification model to differentiate lung cancer patients from healthy controls, and COPD patients from healthy controls. The raw output data from the sensors were pre-processed using the baseline manipulation method before applying to the classifier [3]. From the pre-processed data, five features were extracted based on the previous studies on e-nose technologies in clinical diagnosis. The extracted fea- 
tures were maximum sensor output voltage in the whole measurement time, time of the maximum sensor output voltage, output voltage at a particular time in the whole measurement time, time of the particular sensor output voltage value, and the average sensor output value in the whole measurement period [27]. From each TGS sensor, we extracted five features thereby an overall twenty-five features from the sensor array with five sensors.

Principal component analysis (PCA) was used as the dimensionality reduction technique to reduce the high dimensional vector to a low dimensional vector [11]. After reviewing various papers related to the disease diagnosis using e-nose technology, we concluded that support vector machine (SVM), a supervised learning algorithm gives more accuracy than any of the other supervised and unsupervised learning methods [6], [9], [12], [19]. In this work, we have used SVM for differentiating lung cancer patients from healthy controls and COPD patients from healthy controls.

\section{Results}

\subsection{Sensor characteristics}

The sample sensor array response to the exhaled breath of healthy control (smoker), healthy control (non-smoker), COPD patient, and lung cancer patient are shown in Fig. 3. From the graph, it is clear that the sensor array response voltage to the exhaled breath of cancer and COPD patients is higher than smokers and non-smokers. This variation in the sensor array response is the result of detected VOCs like iso-butane, acetone, carbon monoxide, ethanol, and ammonia with the TGS sensors. When a combination of VOCs is exposed to particular TGS sensors, and when the concentration of that gaseous mixture increases the sensor resistance decreases, and, thereby, the output voltage across the load resistor connected with the sensor increases.

\subsection{Data analysis}

\subsubsection{Traininig phase}

In the training phase of the study 15 lung cancer patients, 12 COPD patients, and 22 healthy controls were included. In Table 3, the details of the study subjects selected for the training phase are shown. $73 \%$ of lung cancer patients, $64 \%$ of healthy controls, and $75 \%$ of COPD patients selected for the study were males because of more frequent occurrence of lung cancer and COPD in males than females. $73 \%$ of the cancer patients and $75 \%$ of COPD patients selected for the study were current or former smokers. The average age of the selected lung cancer patients, COPD patients, and healthy controls are 64,58 , and 55, respec-
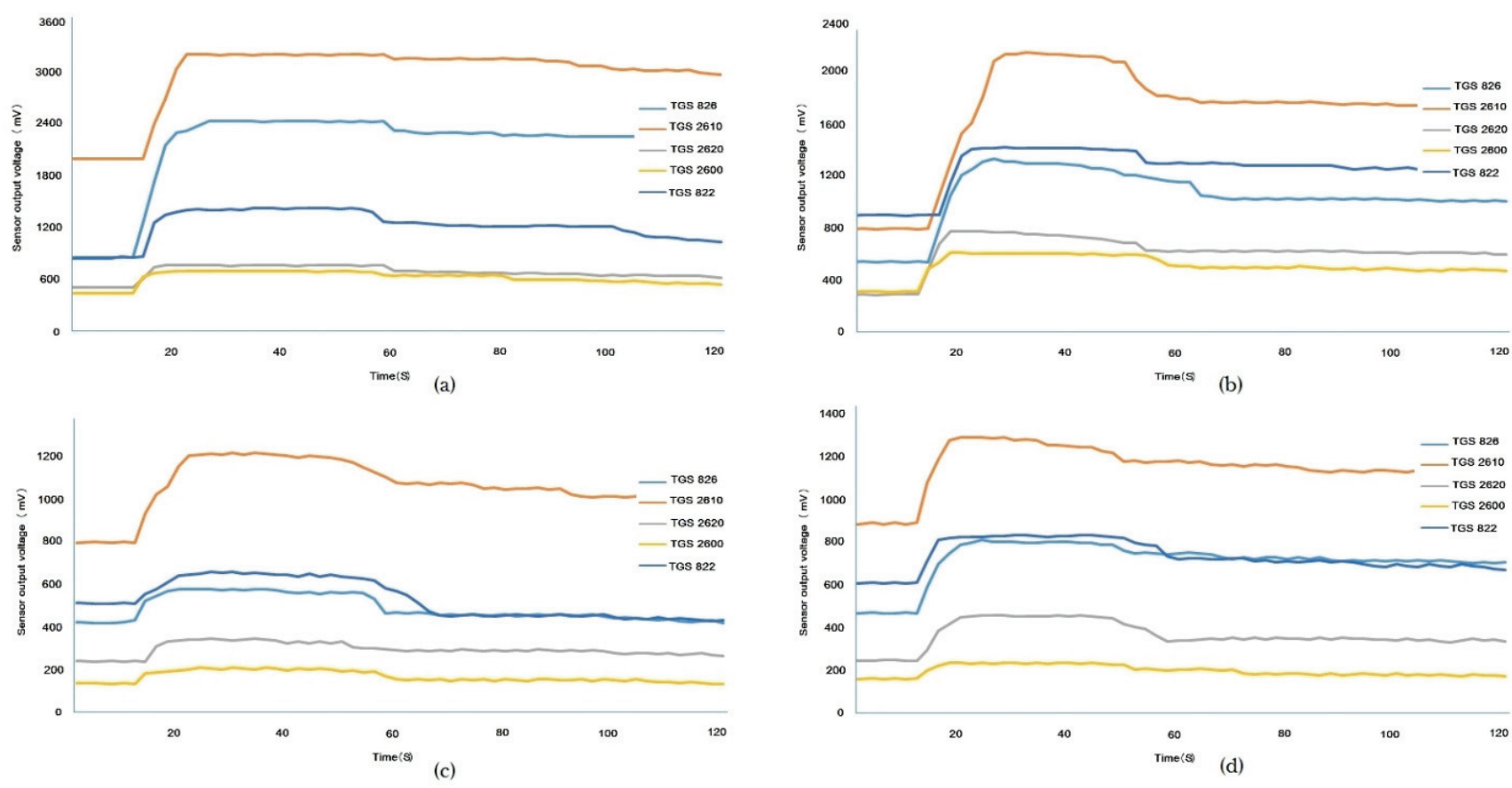

Fig. 3. Sensor array response to: (a) cancer patient, (b) COPD patient, (c) a healthy non-smoker, (d) a healthy smoker 
tively. The average tumor size of cancer affected volunteers is $5.1 \mathrm{~cm}$.

Table 3. Details of the study subjects selected for training set

\begin{tabular}{|l|c|c|c|}
\hline & $\begin{array}{c}\text { Lung } \\
\text { cancer }\end{array}$ & $\begin{array}{c}\text { Healty } \\
\text { control }\end{array}$ & COPD \\
\hline \multicolumn{1}{|c|}{ No. of volunteers } & 15 & 22 & 12 \\
\hline Age [years] & $64 \pm 5$ & $55 \pm 6$ & $58 \pm 4$ \\
\hline Gender & 11 & 14 & 9 \\
Male & 4 & 8 & 3 \\
Female & & & \\
\hline Smoking Status & 4 & 9 & 3 \\
Never smoker & 2 & 9 & 2 \\
Current smoker & 9 & 4 & 7 \\
Former smoker & & & \\
\hline Histology & 14 & & \\
Adenocarcinoma & 1 & \multicolumn{2}{|l}{} \\
Squamous cell carcinoma & $5.1 \pm 1.2$ & & \\
\hline Tumor size [cm] & \multicolumn{3}{|l}{} \\
\hline
\end{tabular}

All the participants enrolled in this study phase were asked to blow the exhaled air to the sensor array system through a tube connected with the system. Four samples were taken from all the participants to generate the training data set and, thereby, creating a training set of 60 lung cancer patient data, 48 COPD patient data, and 88 healthy controls data. Five features were extracted from the pre-processed sensor data and principal component analysis was used for the dimensionality reduction. Figures $4 \mathrm{a}$ and $4 \mathrm{~b}$ show the PCA plot differentiating lung cancer patients from healthy controls and COPD patients from healthy controls, respectively. The classification model for these classes is developed using SVM.

\subsubsection{Validation phase}

In the validation phase of the study design, 12 lung cancer patients, 10 COPD patients, and 17 healthy con-

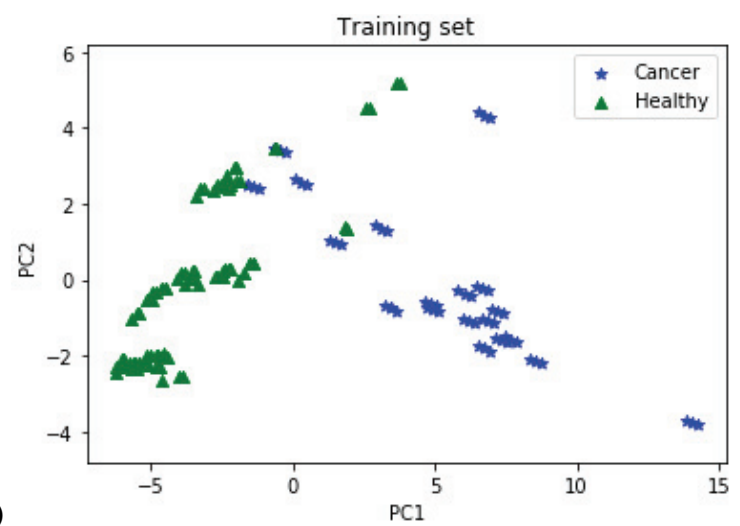

trols have participated. The average age of the lung cancer patients, COPD patients, and healthy controls participated was 65,64 , and 53 , respectively. The details of the study subjects selected for the test set are shown in Table 4. Similar to the training phase of the study, more study subjects were males in all three categories. In the lung cancer patient group, nine of them quit smoking after diagnosed with cancer, two of them had never smoked, and one patient continues his smoking habit. The tumor size of the cancer patients varied from 3.9 to $6.7 \mathrm{~cm}$.

Table 4. Details of the study subjects selected for test set

\begin{tabular}{|l|c|c|c|}
\hline & $\begin{array}{c}\text { Lung } \\
\text { Cancer }\end{array}$ & $\begin{array}{c}\text { Healty } \\
\text { Control }\end{array}$ & COPD \\
\hline No. of volunteers & 12 & 17 & 10 \\
\hline Age [years] & $65 \pm 7$ & $53 \pm 9$ & $64 \pm 6$ \\
\hline Gender & 10 & 10 & 7 \\
Male & 2 & 7 & 3 \\
Female & 2 & 7 & 3 \\
\hline Smoking Status & 1 & 9 & 1 \\
Never smoker & 9 & 1 & 6 \\
Current smoker & 11 & & \\
Former smoker & 1 & & \\
\hline Histology & & & \\
Adenocarcinoma & $5.3 \pm 1.4$ & \multicolumn{2}{|l}{} \\
Squamous cell carcinoma & \multicolumn{3}{|l}{} \\
\hline Tumor size [cm] & & & \\
\hline
\end{tabular}

In this phase of the study, breath samples were collected from all the 39 study subjects four times. The test data for the validation of the prediction model consists of 48 lung cancer patient data, 40 COPD patient data, and 68 healthy controls data. The SVM classifier was able to correctly identify 43 of 48 lung cancer patient samples and 29 of 40 COPD patient samples. The accuracy of discriminating lung cancer patients from healthy control was $88.79 \%$ and COPD patients from healthy controls were $78.70 \%$. The ac-

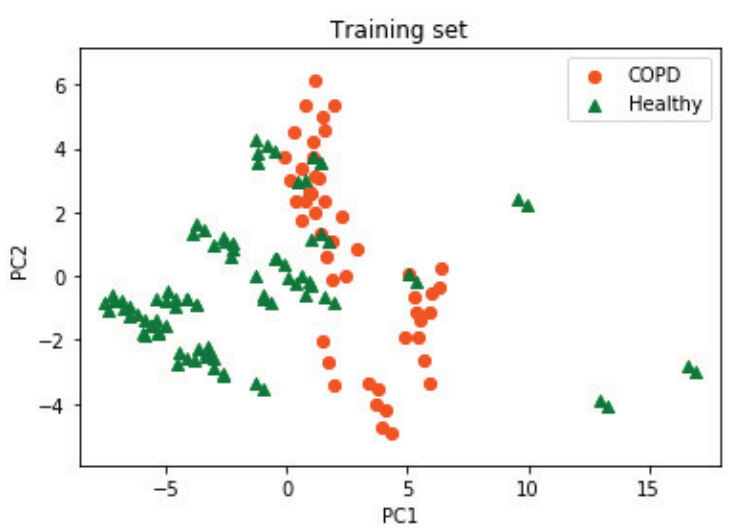

Fig. 4. Training phase PCA plot discriminating: (a) lung cancer patients from healthy controls, (b) COPD patients from healthy controls 
(a)

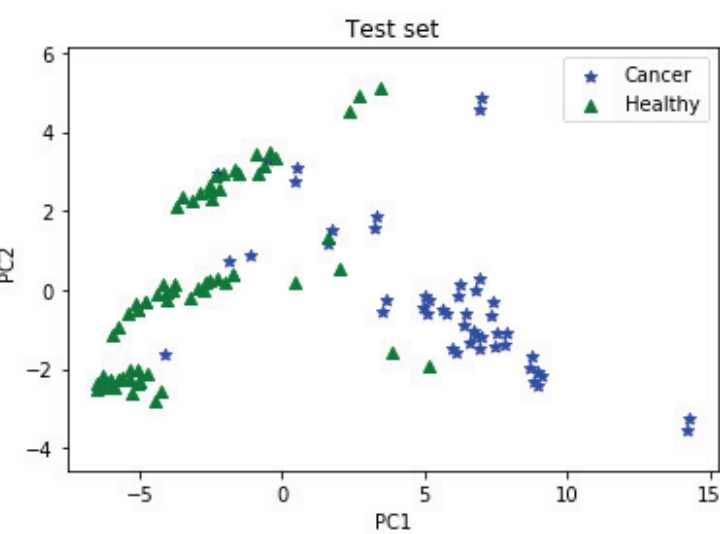

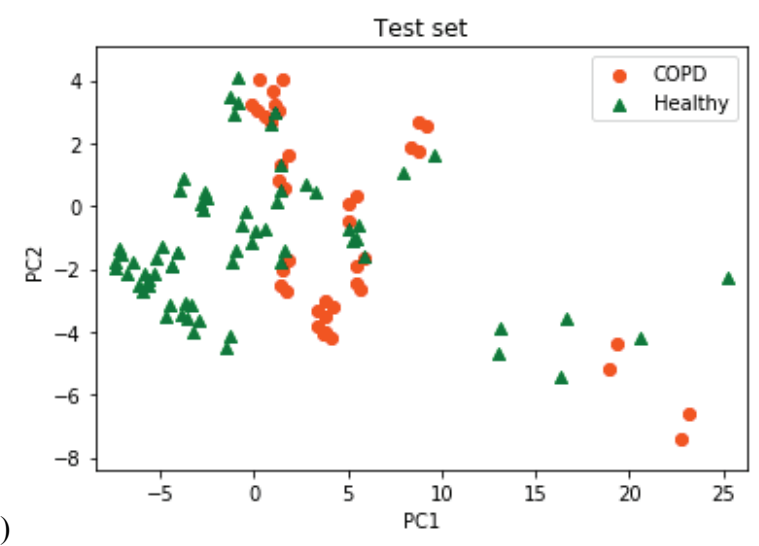

(b)

Fig. 5. Validation phase PCA plot discriminating: (a) lung cancer patients from healthy controls, (b) COPD patients from healthy controls

Table 5. Disease diagnosis parameters

\begin{tabular}{|c|c|c|}
\hline & Lung cancer & COPD \\
\hline Accuracy $[\%]=[(\mathrm{TP}+\mathrm{TN}) /(\mathrm{TP}+\mathrm{TN}+\mathrm{FP}+\mathrm{FN})] \times 100$ & 88.79 & 78.70 \\
\hline Sensitivity $[\%]=[\mathrm{TP} /(\mathrm{TP}+\mathrm{FN})] \times 100$ & 89.58 & 72.50 \\
\hline Specificity $[\%]=[\mathrm{TN} /(\mathrm{TN}+\mathrm{FP})] \times 100$ & 88.23 & 82.35 \\
\hline Positive predictive value $[\%]=[\mathrm{TP} /(\mathrm{TP}+\mathrm{FP})] \times 100$ & 84.31 & 70.73 \\
\hline Negative predictive value $[\%]=[\mathrm{TN} /(\mathrm{TN}+\mathrm{FN})] \times 100$ & 92.30 & 83.58 \\
\hline False negative rate $[\%]=[\mathrm{FN} /(\mathrm{FN}+\mathrm{TP})] \times 100$ & 10.41 & 27.50 \\
\hline False positive rate $[\%]=[\mathrm{FP} /(\mathrm{FP}+\mathrm{TN})] \times 100$ & 11.76 & 17.64 \\
\hline
\end{tabular}

curacy, sensitivity, specificity, positive predictive value, negative predictive value, false-negative rate, and false-positive rate of the electronic nose in the detection of lung cancer and COPD are shown in Table 5. In Figures $5 \mathrm{a}$ and $5 \mathrm{~b}$ the validation phase PCA plot differentiating lung cancer patients from healthy controls and COPD patients from healthy controls are shown, respectively.

\section{Discussion}

Electronic noses (e-noses) are instruments that include an array of chemical sensors and can be used to recognize different odors from the analyzing samples. MOS sensor-based e-noses are nowadays used in various fields including food technology, chemical analysis, and disease diagnosis including diabetes mellitus, COPD, lung cancer and cystic fibrosis [2], [3], [5], [12], [13], [17]. Instead of identifying separate VOCs from gas samples, the MOS-based gas sensors generate a "breath signature" corresponding to different gases. The e-nose system consists of three main parts: a sensor array system, a signal conditioning system, and a pattern recognition system.
Different diseases show different symptoms and the changes in breath VOCs are a great sign of respiratory diseases. There are significant changes in the breath signature for patients with pulmonary diseases like asthma, COPD, cystic fibrosis, and lung cancer. Several studies have been conducted in disease diagnosis using an e-nose system with colorimetric sensors, SAW sensors, PID sensors, QCM sensors, and MOS sensors. Advantages such as good selectivity, fast response, small sensing range, and low power consumption make them suitable for e-nose technology.

In this research, we have developed an e-nose system with an array of five TGS gas sensors TGS 2600, TGS 2610, TGS 2620, TGS 822, and TGS 826, a microcontroller board, and a machine learning algorithm developed in python. This work demonstrates that a MOS-based sensor array with classification algorithms can distinguish lung cancer patients from healthy controls and COPD patients from healthy controls. This system can also discriminate lung cancer patients from COPD patients with acceptable accuracy. The analysis of several samples collected from all the study subjects confirmed this distinction and it shows that the VOC profiles of exhaled breath have significant differences for healthy controls, lung cancer, and COPD. This indicates that e-nose technology 
may be a promising tool for diagnosing pulmonary diseases like asthma, COPD, lung cancer, etc in near future.

This study has a novel approach in selecting the minimum number of sensors that can accurately predict the disease conditions and also selecting the study subjects with almost similar age. We have selected an average age of 64,61 , and 54 for lung cancer patients, COPD patients, and healthy controls, respectively. In previous studies, Chang et al. [6] selected volunteers with an average age of 63 for lung cancer patients and 39 for healthy controls. Oguma et al. [22] studied lung cancer patients with an average age of 66 and healthy controls with an average age of 27, and Marzorati et al. [21] selected the average age of 64 and 27 for lung cancer patients and healthy controls, respectively. Velasquez et al. [28] studied COPD patients and healthy controls with an average age of 72 and 34, respectively. Tan et al. [26] selected volunteers from lung cancer patients, COPD, and healthy controls with an average age of 61,68 , and 49 , respectively. It is preferable to select almost the same age as healthy controls and peoples with diseases for better results. This was a tough task to select the healthy controls that match the age with COPD and cancer patients as most of the peoples in that age contain some sort of respiratory diseases.

\section{Conclusion}

The MOS gas sensor array was able to detect the exhaled breath volatile organic compound profiles produced by the subjects under study. This sensor array system with five TGS MOS gas sensors differentiated lung cancer patients from healthy controls and COPD patients from healthy controls. Here, we demonstrated the adaptability and potentiality of the sensor array system for analyzing the exhaled breath VOC profiles of volunteers with lung cancer, COPD, and with different age, gender, weight, medical history, and smoking habits. In the gas sensor array system, all the five sensors displayed a decrease in sensor resistance in response to the target VOCs and thereby an increase in the sensor output voltage. It is also noted that the TGS sensor gives output response to a composition of breath VOCs instead of a single VOC. The sensor characteristics showed that the sensor output voltages increased in response to the increase in the VOC concentration and we can conclude that the TGS gas sensor output voltage is directly proportional to the volatile organic compounds exposed to it. The e-nose system with the sensor array, microcontroller, and a machine learning algorithm provided an overall accuracy, sensitivity, and specificity of $88.79,89.58$, and $88.23 \%$, respectively, for lung cancer detection, and 78.70, 72.50, and $82.35 \%$ for COPD detection.

Briefly, the sensor array system developed with TGS gas sensors was non-invasive, low cost, and gave a rapid response. Even though the attained results were good, further examinations are essential to enhance the sensor array system, investigate the long run reproducibility and repeatability, and enlarge its relevancy. With a large amount of patient data and the integration of deep learning algorithms for sensor data processing, the developed e-nose system can be used to differentiate between patients with respiratory diseases like asthma, bronchitis, COPD, lung cancer, and healthy controls with $100 \%$ accuracy.

\section{References}

[1] Amann A., Poupart G., Telser S., Ledochowski M., SCHMid A., Mechtcheriakov S., Applications of breath gas analysis in medicine, Int. J. Mass Spectrom., 2004, 239 (2-3), 227-133.

[2] Bannier M.A., van De Kant K.D., Jöbsis Q., Dompeling E., Feasibility and diagnostic accuracy of an electronic nose in children with asthma and cystic fibrosis, J. Breath Res., 2019, 13 (3), 036009.

[3] Blatt R., Bonarini A., Matteucci M., Pattern classification techniques for lung cancer diagnosis by an electronic nose, Computational Intelligence in Healthcare, 2010, 4, 397-423.

[4] Byun H.G., Persaud K.C., Pisanelli A.M., Wound-State Monitoring for Burn Patients Using E-Nose/SPME System, ETRI J., 2010, 32 (3), 440-446.

[5] Byun H.G., Yu J.B., JeOn J.Y., LeE S.Y., Huh J.S., Lim J.O., Exhaled Breath Analysis for Lung Diseases Based on Sensors Array System, Sens. Lett., 2014, 12 (6-7), 1107-1109.

[6] Chang J.E., LeE D.S., BAN S.W., OH J., Jung M.Y., Kim S.H., JHEON S., Analysis of volatile organic compounds in exhaled breath for lung cancer diagnosis using a sensor system, Sensor. Actuat. B-Chem., 2018, 255, 800-807.

[7] Covington J.A., Westenbrink E.W., OuAREt N., HARBord R., Bailey C., O'Connell N., Cullis J., Williams N., Nwokolo C.U., Bardhan K.D., Arasaradnam R.P., Application of a novel tool for diagnosing bile acid diarrhoea, Sensors., 2013, (9), 11899-11912.

[8] D’Amico A., Pennazza G., Santonico M., Martinelli E., Roscioni C., Galluccio G., Paolesse R., Di Natale C., $A n$ investigation on electronic nose diagnosis of lung cancer, Lung Cancer, 2010, 68 (2), 170-176.

[9] Di Natale C., Macagnano A., Martinelli E., Paolesse R., D’Arcangelo G., Roscioni C., D’Amico A., Lung cancer identification by the analysis of breath by means of an array of non-selective gas sensors, Biosens. Bioelectron., 2003, 18 (10), 1209-1218.

[10] Donarelli M., Ottaviano, 2D materials for gas sensing applications: A review on graphene oxide, MoS2, WS2 and phosphorene, Sensors, 2018, 18 (11), 3638.

[11] Duda R.O., Hart P.E., Stork D.G., Pattern classification, John Wiley \& Sons, 2012. 
[12] Dymerski T., GęBicki J., WiśniewsKa P., ŚliwińsKa M., WARDENCKI W., NAMIEŚNIK J., Application of the electronic nose technique to differentiation between model mixtures with COPD markers, Sensors, 2013, 13 (4), 5008-5027.

[13] Guohua H., Yuling W., Dandan Y., Wenwen D., Fuji apple storage time predictive method using electronic nose, Food Anal. Method, 2013, 6 (1), 82-88.

[14] Hakim M., Broza Y.Y., Barash O., Peled N., Phillips M., AMANN A., Haick H., Volatile organic compounds of lung cancer and possible biochemical pathways, Chem. Rev., 2012, 112 (11), 5949-5966.

[15] Hossein-Babaei F., Hosseini-Golgoo S.M., Analyzing the responses of a thermally modulated gas sensor using a linear system identification technique for gas diagnosis, IEEE Sens. J., 2008, 8 (11), 1837-1847.

[16] Itoh T., Matsubara I., Nishibori M., Izu N., Shin W., Calibration gas preparation for non-disposable portable $M O_{x}$, PID, and IER VOC detectors, Sens. Lett., 2012, 10 (3-4), 985-992.

[17] Jolayemi O.S., Tokatli F., Buratti S., Alamprese C., Discriminative capacities of infrared spectroscopy and enose on Turkish olive oils, Eur. Food Res. Technol., 2017, 243 (11), 2035-2042.

[18] Korotcenkov G., Cho B.K., Metal oxide composites in conductometric gas sensors: Achievements and challenges, Sensor. Actuat. B-Chem., 2017, 244, 182-210.

[19] Li W., LiU H., XIE D., He Z., Pi X., Lung cancer screening based on type-different sensor arrays, Sci. Rep-U.K., 2017, 7 (1), 1-12, 2017.

[20] Mannino D.M., Buist A.S., Global burden of COPD: risk factors, prevalence, and future trends, The Lancet, 2007, 370 (9589), 765-773.

[21] Marzorati D., Mainardi L., Sedda G., Gasparri R., Spaggiari L., Cerveri P., A Metal Oxide Gas Sensors Array for Lung Cancer Diagnosis Through Exhaled Breath Analysis, 41st Annual International Conference of the IEEE Engineering in Medicine and Biology Society (EMBC), 2019, 1584-1587.

[22] Oguma T., Nagaoka T., Kurahashi M., Kobayashi N., YAmamori S., Tsuji C., Hayama N., Clinical contributions of exhaled volatile organic compounds in the diagnosis of lung cancer, PloS One, 2017, 12 (4), e0174802.

[23] Pasini P., Powar N., Gutierrez-Osuna R., Daunert S., RODA A., Use of a gas-sensor array for detecting volatile organic compounds (VOC) in chemically induced cells, Anal. Bioanal. Chem., 2004, 378 (1), 76-83.

[24] Pavlou A.K., Magan N., McNulty C., Jones .JM., Sharp D., BROWn J., TURNER A.P., Use of an electronic nose system for diagnoses of urinary tract infections, Biosens. Bioelectron., 2002, 17 (10), 893-899.

[25] Roine A., Veskimäe E., Tuokko A., Kumpulainen P., KOSKIMÄKI J., KEINÄNEN T.A., HÄKKINEN M.R., VEPSÄLÄINEN J., PAavonen T., LeKkala J., LehtiMÄKI T., Detection of prostate cancer by an electronic nose: a proof of principle study, J. Urology, 2014, 192 (1), 230-235.

[26] TAN J.L., Yong Z.X., LiAM C.K., Using a chemiresistorbased alkane sensor to distinguish exhaled breaths of lung cancer patients from subjects with no lung cancer, J. Thorac. Dis., 2016, 8 (10), 2772.

[27] Voss A., Baier V., Reisch R., von Roda K., Elsner P., Ahlers H., Stein G., Smelling renal dysfunction via electronic nose, Ann. Biomed. Eng., 2005, 33 (5), 656-660.

[28] Velásquez A., Durán C.M., Gualdron O., Rodríguez J.C., MANJARRes L., Electronic nose to detect patients with COPD from exhaled breath, [in:] AIP Conference Proceedings, 2009, Vol. 1137, No. 1, 452-454.

[29] Wojnowski W., Dymerski T., GęBicki J., NAmieŚnik J., Electronic noses in medical diagnostics, Curr. Med. Chem., 2019, 26 (1), 197-215. 